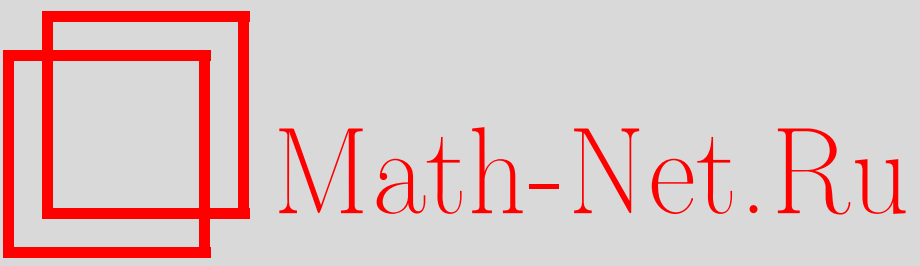

Л. О. Чехов, Н. В. Пузырникова, Интегрируемые системы на графах, УМН, 2000, том 55, выпуск 5, 181-182

DOI: https://doi.org/10.4213/rm329

Использование Общероссийского математического портала Math-Net.Ru подразумевает, что вы прочитали и согласны с пользовательским соглашением

http://www.mathnet.ru/rus/agreement

Параметры загрузки:

IP: 54.209 .52 .79

26 апреля 2023 г., 14:45:02 


\title{
ИНТЕГРИРУЕМЫЕ СИСТЕМЫ НА ГРАФАХ
}

\author{
Л.О. Чехов, Н. В. ПузырниковА
}

Статистические и интегрируемые системы на граффах представляют собой удобный объект исследования. Спектральные задачи для операторов второго порядка допускают целый ряд точных утверждений [1]-[3]. В то же время нелинейные интегрируемые системы были построены только на решетках $\mathbb{Z}\left((L, A)\right.$-пары типа цепочки Тоды) и $\mathbb{Z}^{2}((L, A, B)$-тройки), см. [4]. В недавней работе Кричевера и Новикова [5] на графе $\Gamma_{3}$ (бесконечное однородное дерево с вершинами валентности три) была построена деформация произвольного оператора $L$ четвертого порядка в виде $(L, A, B)$-тройки, $\dot{L}=L A-B L$, которая сохраняет спектральные данные нулевого уровня $L \psi=0$. (Никаких локальных изоспектральных деформаций операторов $L$ второго порядка на деревьях обнаружить не удалось.)

В этой работе интегрируемая система Кричевера-Новикова обобщается на случай произвольного граффа $\Gamma$. Рассматриваются операторы $L$, действующие на пространстве функций $\psi_{P}$, зависяших от вершин графа $\Gamma$. Расстояние $d\left(X_{1}, X_{2}\right) \in \mathbb{Z}_{+} \cap\{0\}$ на дереве без циклов измеряется в длинах ребер, входящих в путь, соединяющий вершины $X_{1}$ и $X_{2}$. Порядком уравнения $L \psi=0$, где $(L \psi)_{P}=\sum_{X} b_{P, X} \psi_{X}$, назьвается максимальный диаметр $\max _{P} d\left(X_{1}, X_{2}\right): b_{P, X_{1}} \neq 0$, $b_{P, X_{2}} \neq 0$ или $b_{X_{1}, X_{2}} \neq 0$. Рассмотрим сначала произвольный граф без петель.

Теорема. Вещественный самосопряженный оператор L четвертого порядка на графе Г без петель обладает изоспектральной деформачией одного уровня энергии $L \psi=0$ в виде $(L, A, B)$-тройки $\left(B=A^{t}\right)$

$$
\dot{L}=L A+A^{t} L
$$

где $A$ - оператор второго порядка,

$$
(A \psi)_{X}=\sum_{X^{\prime}: d\left(X, X^{\prime}\right)=1} a_{X, X^{\prime}} \psi_{X^{\prime}}+s_{X} \psi_{X}
$$

тогда и только тогда, когда оператор $L$,

(3) $(L \psi)_{X}=\sum_{X^{\prime \prime}: d\left(X, X^{\prime \prime}\right)=2} b_{X, X^{\prime \prime}} \psi_{X^{\prime \prime}}+\sum_{X^{\prime}: d\left(X, X^{\prime}\right)=1} r_{X, X^{\prime}} \psi_{X^{\prime}}+\rho_{X} \psi_{X}$,

такой, что все $b_{X, X^{\prime \prime}}>0$, представим в виде

$$
L=Q^{t} Q+u_{X}
$$

где $Q$ - оператор второго порядка,

$$
(Q \psi)_{X}=\sum_{X^{\prime}: d\left(X, X^{\prime}\right)=1} q_{X, X^{\prime}} \psi_{X^{\prime}}+v_{X} \psi_{X}
$$

При этом коэффициенты $q_{X, X^{\prime}}$ определены однозначно, а коэффициенты $v_{X}$ содержат свободный параметр - значение $v_{X_{0}}$ в некоторой избранной точке $X_{0}$.

(На дереве $\Gamma_{3}$ произвольный оператор четвертого порядка $L(3)$ допускает представление (4), а потому имеет изоспектральную деформацию (1) [5].)

Из требования того, что деформация (1) оператора $L$ с помощью оператора $A$ второго порядка представляет собой снова оператор четвертого порядка, следует, что для любых четырех

Работа выполнена при частичной поддержке Российского фонда фундаментальных исследований (грант № 99-01-00151). 
различных вершин $X_{1}, \ldots, X_{4}$ таких, что $d\left(X_{i}, X_{j}\right)=2, i \neq j$ (заметим, что тогда существует единственная вершина $X_{0}$ такая, что $\left.d\left(X_{i}, X_{0}\right)=1, i=1, \ldots, 4\right)$, коэффициенты $b_{X_{i}, X_{j}}$ (всегда предполагающиеся симметричными по своим индексам, см. (3)) должны удовлетворять соотношениям

$$
b_{X_{1}, X_{2}} b_{X_{3}, X_{4}}=b_{X_{1}, X_{3}} b_{X_{2}, X_{4}}=b_{X_{1}, X_{4}} b_{X_{2}, X_{3}} .
$$

Из условий (6) следует взаимно однозначное соответствие между $q_{X_{i}, X_{0}}$ и $b_{X_{i}, X_{j}}$, где $X_{i}$, $i=1, \ldots, n,-$ все вершины, отстояшие от вершины $X_{0}$ на единичное расстояние. В одну сторону, $b_{X_{i}, X_{j}}=q_{X_{i}, X_{0}} q_{X_{j}, X_{0}}$. В другую сторону, с учетом соотношений (6),

$$
b_{X_{k}, X_{l}}=\frac{b_{X_{1}, X_{k}} b_{X_{1}, X_{l}} b_{X_{2}, X_{3}}}{b_{X_{1}, X_{2}} b_{X_{1}, X_{3}}} \text { при } k, l>1, \quad k \neq l,
$$

$$
q_{X_{k}, X_{0}}=\frac{b_{X_{1}, X_{k}} \sqrt{b_{X_{2}, X_{3}}}}{\sqrt{b_{X_{1}, X_{2}} b_{X_{1}, X_{3}}}}, k>1, \quad q_{X_{1}, X_{0}}=\sqrt{\frac{b_{X_{1}, X_{2}} b_{X_{1}, X_{3}}}{b_{X_{2}, X_{3}}}} .
$$

Коэффициенты $a_{X, X^{\prime}}$ оператора деформации (2) выражаются однозначным образом (с точностью до общей мультипликативной константы) через $q_{X, Y}$. Для любых трех точек $X_{1}, X_{2}, X_{3}$ таких, что $d\left(X_{i}, X_{i+1}\right)=1, i=1,2$, имеем

$$
\frac{a_{X_{1}, X_{2}}}{q_{X_{2}, X_{1}}}=\frac{a_{X_{1}, X_{3}}}{q_{X_{3}, X_{1}}} \text { и } a_{X_{3}, X_{2}}=-a_{X_{2}, X_{1}} \frac{q_{X_{2}, X_{3}}^{2}}{q_{X_{1}, X_{2}} q_{X_{3}, X_{2}}}
$$

(в частности, при $X_{1}=X_{3}$ получим $a_{X_{1}, X_{2}}=-a_{X_{2}, X_{1}} q_{X_{2}, X_{1}}^{2} / q_{X_{1}, X_{2}}^{2}$ ).

ЗАмечАниЕ 1 . Оператор деформации (2) задается также в терминах коцикла

$$
\chi_{X_{1}, X_{2}}=-\frac{\prod_{i=1}^{n_{2}}\left(b_{X_{1}, X_{i}^{\prime}}\right)^{1 / n_{2}}}{\prod_{j=1}^{n_{1}}\left(b_{X_{j}^{\prime \prime}, X_{2}}\right)^{1 / n_{1}}},
$$

обобшающего коцикл, предложенный в [5]. Здесь $X_{1}$ и $X_{2}$ - две соседние вершины с соответствующими полными валентностями $n_{1}+1$ и $n_{2}+1$, а $\left\{X_{j}^{\prime \prime}\right\}$ и $\left\{X_{i}^{\prime}\right\}$ - наборы вершин, соседних соответственно вершинам $X_{1}$ и $X_{2}$ и не совпадающих с $X_{1}, X_{2}$.

Явные формулы для деформации имеют вид:

$$
\begin{aligned}
& \dot{b}_{X_{i}, X_{j}}=r_{X_{i}, X_{0}} a_{X_{0}, X_{j}}+a_{X_{0}, X_{i}} r_{X_{0}, X_{j}}+b_{X_{i}, X_{j}}\left(s_{X_{i}}+s_{X_{j}}\right), \\
& \dot{r}_{X_{1}, X_{2}}=\sum_{X^{\prime \prime}} b_{X_{1}, X^{\prime \prime}} a_{X^{\prime \prime}, X_{2}}+\sum_{X^{\prime}} b_{X_{2}, X^{\prime}} a_{X^{\prime}, X_{1}}+\rho_{X_{1}} a_{X_{1}, X_{2}}+\rho_{X_{2}} a_{X_{2}, X_{1}} \\
& +r_{X_{1}, X_{2}} s_{X_{2}}+r_{X_{2}, X_{1}} s_{X_{1}} \\
& \dot{\rho}_{X}=\sum_{X^{\prime}} r_{X, X^{\prime}}\left(a_{X^{\prime}, X}+a_{X, X^{\prime}}\right)+2 \rho_{X} s_{X} .
\end{aligned}
$$

Лемма. Деформация (8) с учетом (7) сохраняет условия (6) (и, следовательно, условие факторизации (4)).

ЗАмечАниЕ 2. На произвольном графе (с петлями) факторизация (4) и, одновременно, деформация (1) существуют, если и только если коцикл $\chi_{X_{1}, X_{2}}$ когомологичен нулю.

\section{СПИСОК ЛИТЕРАТУРЫ}

[1] Новиков С. П. // УМН. 1997. Т. 52. №6. С. 178-179. [2] Novikov S. P. Schrödinger operators on graphs and symplectic geometry // Специалњный выпуск института Филдса (Торонто, 1999), посв. 60-летию В. И. Арнольда. [3] Чехов Л. О. // УМН. 1999. Т. 54. №6. С. 109-148. [4] Новиков С.П., Дынников И. А. // УМН. 1997. Т. 52. № 5. С. 175-234. [5] Кричевер И. М., Новиков С. П. // УМН. 1999. Т. 54. №6. С. 149-150. 